Sharif University of Technology
Scientia Iranica
Transactions E: Industrial Engineering
http://scientiairanica.sharif.edu

\title{
A multi-objective two-echelon newsvendor problem with limited budget and capacity
}

\author{
M. Yazdi ${ }^{a, *}$ and S.H.R. Pasandideh ${ }^{b}$ \\ a. Department of Industrial Engineering, Faculty of Industrial and Mechanical Engineering, Qazvin Branch, Islamic Azad \\ University, Qazvin, P.O. Box 34185-1416, Iran. \\ b. Department of Industrial Engineering, Faculty of Engineering, Kharazmi University, Tehran, Iran.
}

Received 23 February 2019; received in revised form 8 November 2019; accepted 3 March 2020

\author{
KEYWORDS \\ Newsvendor problem; \\ Inventory \\ management; \\ Nonlinear \\ programming; \\ SUMT; \\ SD.
}

\begin{abstract}
In this paper, a two-echelon newsvendor problem is considered. Many real-life spheres including fashion, food industries, and healthcare services run into problems similar to newsvendor problem. Our problem is determining inventory levels in order to optimize the profit and service level in selling a product. This product is made up of several raw materials. Only the distribution of demand is known and the hot season of selling the product is just a short period; after that, the price of the product drops dramatically. The storage space and initial budget are limited. The problem is modeled and solved as an unconstrained nonlinear optimization problem using two nonlinear techniques: the Sequential Unconstrained Minimization Technique (SUMT) and Steepest Descent (SD).

(C) 2022 Sharif University of Technology. All rights reserved.
\end{abstract}

\section{Introduction}

The newsvendor model (NVM) is a well-known technique of inventory management in operations research that has been initially developed for encountering uncertain demand for perishable products when the period of selling is short. Various NVM extensions and applications exist in the literature [1].

Although the application of the problem in manufacturing is usual, in recent years, some authors have employed the NVM for healthcare services. Sariyer [2] used the NVM for determining capacities at the call center of emergency medical services. Olivares et al. [3] employed maximum likelihood estimations for cost parameters and applied the NVM. They then presented an econometric model for reserving cardiac surgeries in operating rooms. Wachtel and Dexter [4] applied

*. Corresponding author. Tel.: +982833665275 E-mail addresses: yazdi_maryam@yahoo.com (M. Yazdi); shr_pasandideh@khu.ac.ir (S.H.R.Pasandideh)

doi: $10.24200 /$ sci. 2020.52992 .3001 the NVM to the case of uncertainty in demands and utilized the model for admitting patients in operating rooms according to medical specialties. Abedini et al. [5] used the NVM for planning events in the operating room.

Many extensions of the classic model have been developed to adapt NVM to real-world problems. Khouja [6] presented a taxonomy of these extensions. Sundar et al. [7] considered the NVM when the distribution of demand was unknown and only its mean and variance were known. Subrata [8] investigated a special NVM in which the price of inventory after the selling period was a decision variable. Sainathan and Groenevelt [9] managed to analyze a supply chain with various assumptions like buyback and discount using the NVM. Tekin and Özekici [10] dealt with a case in which there was risk in both demand and supply by using the mean variance framework in the NVM. Pal et al. [11] studied a special NVM with an unknown distribution function of demand, where only the mean and variance of the distribution of demand were known. They assumed that the holding cost was a nonlinear function of ordering quantity and any 
increase or decrease in the holding cost was in line with the order quantity. Shi et al. [12] presented a dynamic risk-averse NVM in order to overcome time inconsistency of formal risk measurements. Wang et al. [13] compared multi ordering versus single ordering in a dynamic NVM, when the newsvendor updated the forecast of demand dynamically. Abrudan et al. [14] studied the role of NVM in drop-shipping activities. Bieniek introduced a special NVM with the aim of maximizing customer satisfaction. This objective function is interpreted as the probability of exceeding a pre-specified target profit [15]. Kim et al. [16] studied multi-period NVM and formulated it as a multi-stage stochastic programming. An NVM with correlated demand in which period-to-period demand is dependent was given in $[17,18]$. Xu et al. [19] extended an NVM with the criterion of minimizing opportunity loss. Khouja et al. [20] examined an NVM in case that some customers only buy products at a discount. Dai and Meng [21] studied NVM when a risk-averse newsvendor makes decisions on pricing, marketing, and ordering under conditional-value-at-risk measures. Pasandideh et al. [22] developed an NVM in the case of two-echelon and a limited budget. Guler et al. [23] considered a special case in which two competitors use NVM for their firms and keep their cost private and then, calculated the equilibrium of the system based on some game theory concepts. Mohammadivojdan and Geunes [24] considered a special NVM in which a seller could obtain a product from various suppliers; each supplier has its own price and proposes quantitybased discounts because of the limited storage. Zhang et al. [25] focused on NVM in a multi-period problem when the distribution of demand is unknown. Adhikary et al. [26] considered the distribution-free version of the NVM when the demand is supposed as fuzzy-random variable. Watt and Vazquez [27] investigated the NVM when salvage could be sold back to the wholesaler.

This study extended the work of Pasandideh et al. [22] and considered a two-echelon NVM in a single period with two criteria. In this system, a product is produced and sold at a regular price during an interval and after that interval, all the stock is sold at a discount. Therefore, the producer encounters two phases for inventory cycle. In the first phase, production is performed; in the second phase, when there is not any production, only stocks can be sold. This system fits with two-echelon inventory systems [28]. Our main contribution is twofold: utilizing nonlinear techniques and mixing the exact methods, the Sequential Unconstrained Minimization Technique (SUMT), and Steepest Descent (SD) from non-linear programming techniques in solving NVM. To our knowledge, this solution method has rarely been used in previous NVM literature. Table 1 depicts some recent literature on the newsvendor problem. Our second contribution is considering profit of sale as the objective of producers with the service level as the objective of customers. In this way, we consider the benefit of both producer and customer as two different stockholders of the problem in our objective function.

Our problem is modeled under stochastic demand with the assumption that demand has a specific distribution. Because the objective functions and constraints are differentiable, we are able to employ the SUMT and SD techniques for presenting a solution method. In our method, each objective function is separately optimized considering the constraints. Finally, these two objective functions are combined with each other using the global criterion method by assigning weights to the initial objective functions. The resulting objective function is solved under the constraints of the model using SUMT and SD techniques.

This paper is organized as follows: Section 2 provides an overview of the problem definitions. Section 3 discusses the solution approach. Section 4 presents some computational results. Finally, Section 5 presents conclusions and some outlines for future works.

\section{Problem description}

A producer wants to produce a fashionable product that is made up of several raw materials. The hot season of selling the product is limited and after that, the demand declines dramatically. All raw materials and products that remain after the hot season will be sold with discounts. The producer has a limited budget and encounters limitation on space of saving materials. Only the distribution of the demand is known. The producer prepares some products before the selling season, but only some fraction of excess demand can be produced during the selling season. The production process has wastage. There are costs for lost sales and saving materials. The amount of buying raw materials and producing products before the season of selling should be determined.

The objective of the problem is maximizing the profit of sale and service level. Other details of the problem are described in the following.

\subsection{Assumptions}

- There is only one opportunity or a single period to sell the final product at a normal price, and when this period is over, all that remains (i.e., raw materials and the finished products) will be sold at discounted prices;

- The raw materials can only be purchased before the beginning of the selling period. In addition, some quantities of the final products can be produced before this period. These values are unknown and should be determined;

- The demand for the finished product during the 
Table 1. A summary of current newsvendor model (NVM) literature.

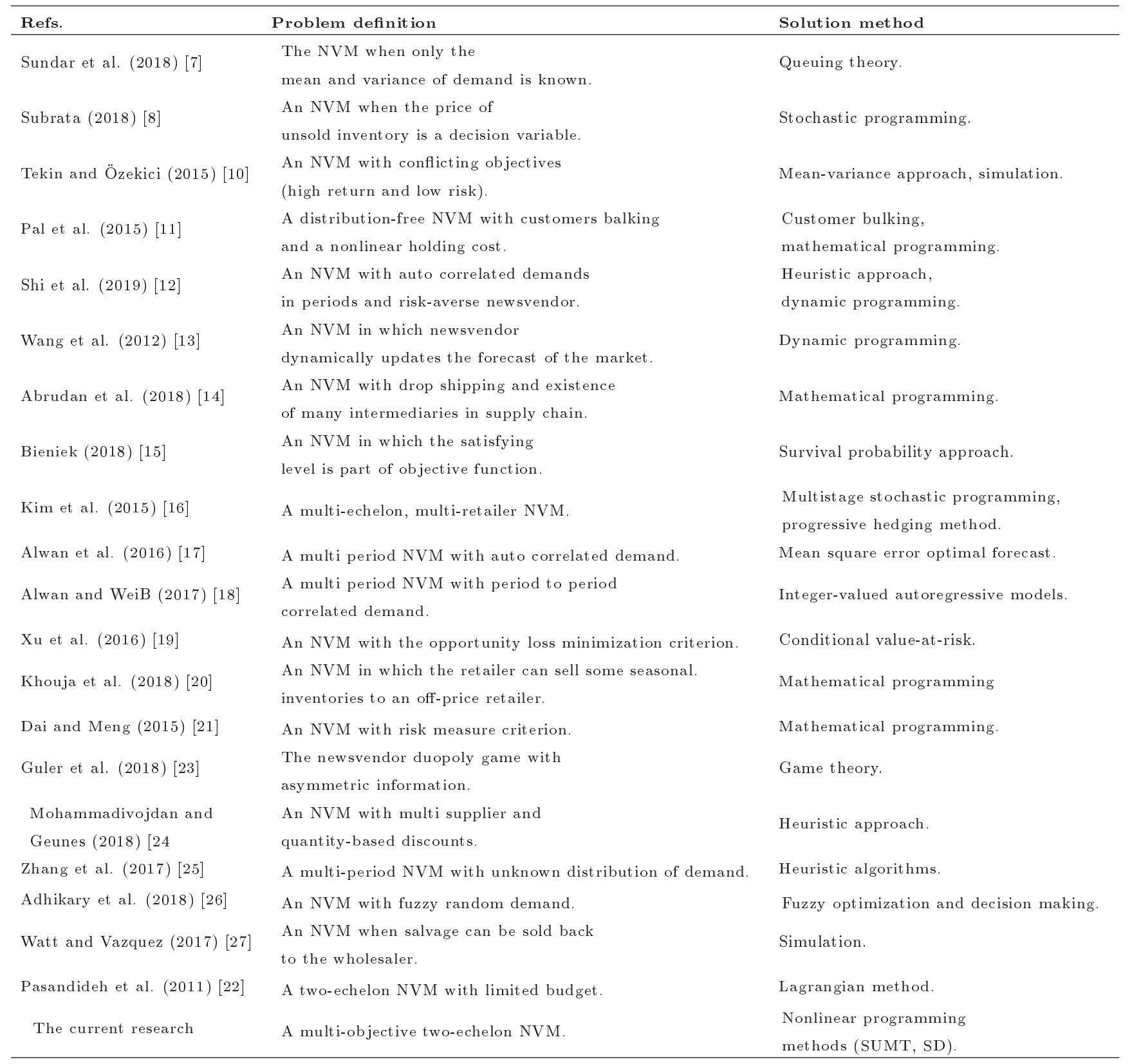

selling period is a random variable with a specific distribution. If the demand is higher than the initial inventory of the final product, a specific fraction of unsatisfied demands will be backordered and produced during this period using the initial inventory of raw materials. The backordered demand would be sold at a normal price. However, the remaining unsatisfied demand will go to the eager competitors, resulting in a specific cost of lost sales;

- The production process has a specific wastage rate and this wastage is immediately sold out for a specific price per unit;

- The cost of transforming raw materials to the final product is a multiplier of the amount of the final product per unit;

- There is a limited predetermined budget;

- The amount of storage space is limited and each unit of raw materials and the final product occupies a specific amount of space;

- The holding costs of the finished product and raw materials for the entire period are specific amounts, and the holding costs of the finished products and raw materials for a fraction of the period are negligible;

- The service level is considered as the percentage of demand that is satisfied during the period. 


\subsection{Definition of parameters}

$Q R_{i} \quad$ The quantity of the $i$ th raw material purchased before the selling period

QR The minimum amount of various raw materials: $Q R=\min \left(Q R_{1}, Q R_{2}, \ldots, Q R_{n}\right)$

$Q S \quad$ The quantity of the final products produced before the selling period

$R \quad$ The normal selling price of each unit of the finished product during the period

$l_{w} \quad$ The selling price of each unit of the wastage

$C_{i} \quad$ The purchasing price of each unit of the $i$ th raw material

$C^{\prime} \quad$ The cost of transforming raw materials into one unit of the finished product

$V S \quad$ The space occupied by each unit of the finished product

$V R_{i} \quad$ The space occupied by each unit of the $i$ th raw material

$\beta \quad$ Wastage rate of the production process

$h_{i} \quad$ The cost of holding each unit of the $i$ th raw material for the entire period

$H \quad$ The cost of holding each unit of the finished product for the entire period

$L_{i} \quad$ The selling price of each unit of the $i$ th raw material after the end of the period

$L^{\prime} \quad$ The selling price of each unit of the finished product after the end of the period

$\alpha \quad$ A fraction of customers who can wait for their demands to be fulfilled by production during the period

$\pi \quad$ The cost of lost sales for each unit of the final product

$D \quad$ A random variable that determines the amount of demand during the period

$f_{d}(d) \quad$ The probability density function of the demand during the period

$F_{D}(d) \quad$ The cumulative distribution function of the demand during the period

$B \quad$ Total available budget

I Total available storage space

U Total profit

$\bar{U} \quad$ The expected total profit

$S L \quad$ The service level

$\overline{S L} \quad$ The expected service level

2.3. Possible scenarios in problem modeling

Without loss of generality, the relationships between raw materials and the finished product are considered one by one. This means that to produce each unit of the finished product, one unit of each kind of the raw materials is consumed. For this reason, $Q R$ replaces all values of $Q R_{i}$ [22].

The production process has $\beta$ percent of wastage. Therefore, production $Q S$ units of the finished product consume $(1+\beta) Q S$ units of raw materials and give $\beta^{*} Q S$ units of wastage. Because the amount of demand is a random variable, three scenarios may be encountered during the period.

\subsubsection{The first scenario}

The first scenario takes place when the amount of demand is less than the initial inventory level of the finished product, i.e., $D \leq Q S$. In this case, no further production is required during the period, and it is possible that some amounts of the initial finished product will remain unsold. Obviously, all demands will be satisfied and the service level will be one hundred Eq. (1):

$$
S L_{1}=100 \text {. }
$$

For calculating the total profit $U_{1}$, all the costs should be subtracted from all the revenues.

The revenues are of three types: (1) The income from selling the finished product at a normal price during the period of demand $r D ;(2)$ The income from selling wastage derived from the production of the $Q S$ units of the finished product before the beginning of the period $l_{w}(\beta Q S)$; and (3) The income from selling the remaining raw materials $\sum_{i}^{n} L_{i} Q R$ and the remaining finished product $L^{\prime}(Q S-D)$ at a discounted price.

Costs are of three types: (1) The purchasing cost of raw materials (i.e., the initial inventory of raw materials $Q R$ and the raw materials used for the initial production of $(1+\beta) Q S$ units of the finished product); (2) The cost of the initial production of the finished product; and (3) The holding costs of all the raw materials and the unsold finished products during the period.

$$
\begin{aligned}
U_{1}= & r D+\sum_{i=1}^{n} L_{i} Q R+L^{\prime}(Q S-D) \\
& -\left[\sum_{i=1}^{n} C_{i} Q R+\left(C^{\prime}+\sum_{i=1}^{n} C_{i}\right)(1+\beta) Q S\right. \\
& \left.-l_{w}(\beta Q S)+\sum_{i=1}^{n} h_{i} Q R+h^{\prime}(Q S-D)\right] .
\end{aligned}
$$

\subsubsection{The second scenario}

The second scenario takes place when the amount of demand is greater than the initial inventory level of the finished product, i.e., $D \geq Q S$. In this scenario, by using the initial inventory of raw materials and producing the finished product during the period, we can satisfy all demands of loyal customers. These 
customers prefer waiting for the production to buying from the competitors.

If we consider the wastage derived from the production of $\alpha(1+\beta)(D-Q S)$ units of the finished product during the period, the initial inventory of raw materials is as follows:

$$
a(1+\beta)(D-Q S) \leq Q R
$$

After simplifications, the demand value in this scenario is given as follows:

$$
Q S \leq D \leq Q S+\frac{Q R}{\alpha(1+\beta)} .
$$

The service level in this scenario is the percentage of demand which is satisfied as follows:

$$
S L_{2}=100 \frac{(Q S+\alpha(D-Q S)}{D} .
$$

Here, the total profit $U_{2}$ is calculated as in the previous scenario. Revenues are of three types: (1) The income from selling $(Q S+\alpha(D-Q S))$ units of the finished product at a normal price during the period; (2) The income from selling the wastage associated with the initial production and with the production during the selling period, i.e., $l_{w}(\beta Q S)+l_{w}(\alpha \beta(D-Q S))$; and (3) The income from selling the remaining raw materials $Q R-\alpha(1+\beta)(D-Q S)$ at a discounted price.

Costs are of four types: (1) The purchasing cost of raw materials before the beginning of the period (i.e., the initial inventory of raw materials $Q R$ and the raw materials used for initial production of $(1+\beta) Q S$ units of the finished product); (2) The cost of the initial production of the finished product and the cost of the production during the period; (3) The cost of lost sales $(1+\alpha)(D-Q S)$; and finally, (4) The holding costs of the remaining raw materials to the end of the period $Q R-\alpha(1+\beta)(D-Q S)$.

$$
\begin{aligned}
U_{2}= & r(Q S+\alpha(D-Q S))+\sum_{i=1}^{n} L_{i}(Q R \\
& -\alpha(1+\beta)(D-Q S))+l_{w} \alpha \beta(D-Q S) \\
& +l_{w}(\beta Q S)-\left[\sum_{i=1}^{n} C_{i} Q R\right. \\
& +\left(C^{\prime}+\sum_{i=1}^{n} C_{i}\right)(1+\beta) Q S+C^{\prime} \alpha(1+\beta) \\
& (D-Q S)+(1-\alpha)(D-Q S) \pi \\
& +\sum_{i=1}^{n} h_{i}(Q R-\alpha(1+\beta)(D-Q S)] .
\end{aligned}
$$

\subsubsection{The third scenario}

The third scenario is somewhat similar to the second one in that the amount of demand is greater than the initial inventory of the finished product $(D \geq Q S)$. However, the difference is that the initial inventory of raw materials is not sufficient to satisfy all loyal customers. In other words, the initial inventory of raw materials is less than $\alpha(1+\beta)(D-Q S)$ or $D \geq$ $Q S+\frac{Q R}{\alpha(1+\beta)}$.

The service level in this case or the percentage of the satisfied demand is given below:

$$
S L_{3}=100 \frac{Q S+\frac{Q R}{(1+\beta)}}{D} .
$$

For the total profit $U_{3}$, revenues are of two types: (1) The income from selling the $Q S+\frac{Q R}{(1+\beta)}$ amount of the finished product at a normal price during the period; (2) The income from selling the wastages of the initial production and of the production during the period $l_{w}(\beta Q S)+l_{w}(\beta Q R)$.

Costs are of three parts: (1) The purchasing cost of raw materials before the beginning of the period (consisting of raw materials used for the initial production $(1+\beta) Q S$ and the initial inventory of raw materials $Q R$ ); (2) The cost of production of the initial number of the finished products and the cost of production during the period; and (3) The cost of the amount $D-Q S-\frac{Q R}{(1+\beta)}$ of lost demand.

$$
\begin{aligned}
U_{3}= & r\left(Q S+\frac{Q R}{(1+\beta)}\right)+l_{w}(\beta Q S)+l_{w}(\beta Q R) \\
& -\left[\sum_{i=1}^{n} C_{i} Q R+\left(C^{\prime}+\sum_{i=1}^{n} C_{i}\right)(1+\beta) Q S\right. \\
& \left.+C^{\prime} Q R+\left(D-Q S-\frac{Q R}{(1+\beta)}\right) \pi\right]
\end{aligned}
$$

\subsection{The mathematical model}

The profit and the service level are dependent on the value of the random variable demand. In order to model the problem, the expected value of this function should be considered.

The expected service level $(\overline{S L})$ is as follows:

$$
\begin{aligned}
\overline{S L}= & \int_{0}^{\infty} S L(Q R, Q S, D) f_{D}(d) d(D) \\
\overline{S L}= & \int_{0}^{Q S} S L_{1} f_{D}(d) d(D)+\int_{Q S}^{Q S+\frac{Q R}{\alpha(1+\beta)}} \\
& S L_{2} f_{D}(d) d(D)+\int_{Q S+\frac{Q R}{\alpha(1+\beta)}}^{\infty} S L_{3} f_{D}(d) d(D) .
\end{aligned}
$$

The expected profit $(\bar{U})$ is: 


$$
\begin{aligned}
\bar{U}= & \int_{0}^{\infty} U(Q R, Q S, D) f_{D}(d) d(D), \\
\bar{U}= & \int_{0}^{Q S} U_{1} f_{D}(d) d(D)+\int_{Q S}^{Q S+\frac{Q R}{\alpha(1+\beta)}} U_{2} f_{D}(d) d(D) \\
& +\int_{Q S+\frac{Q R}{\alpha(1+\beta)}}^{\infty} U_{3} f_{D}(d) d(D) .
\end{aligned}
$$

We assume that the demand is a continuous random variable with a uniform distribution function at the interval $\left[d_{\min }, d_{\max }\right]$, where $d_{\min }$ and $d_{\max }$ are nonzero and positive real numbers.

The objective function with this assumption changes as follows:

$$
\begin{aligned}
\overline{S L}= & \int_{d_{\min }}^{Q S} S L_{1}\left(\frac{1}{\left(d_{\max }-d_{\min }\right)}\right) d(D)+\int_{Q S}^{Q S+\frac{Q R}{\alpha(1+\beta)}} \\
& S L_{2}\left(\frac{1}{\left(d_{\max }-d_{\min }\right)}\right) d(D)+\int_{Q S+\frac{Q R}{\alpha(1+\beta)}}^{d \max } \\
& S L_{3}\left(\frac{1}{\left(d_{\max }-d_{\min }\right)}\right) d(D) .
\end{aligned}
$$

After substituting $S L_{1}, S L_{2}$, and $S L_{3}$ in Eq. (11) for $\overline{S L}$ and through integration, the expected service level or the first objective function is simplified as follows:

$$
\begin{aligned}
\overline{S L}= & \left(\frac{100}{\left(d_{\max }-d_{\min }\right)}\right)\left(Q S-d_{\min }+\frac{Q R}{(1+\beta)}\right. \\
& -\left(\alpha Q S+\frac{Q R}{(1+\beta)}\right) \ln \left(Q S+\frac{Q R}{(1+\mid ! \beta)}\right) \\
& -(1-\alpha) Q S \ln (Q S)+\left(Q S+\frac{Q R}{(1+\beta)}\right) \\
& \left.\ln \left(d_{\max }\right)\right) .
\end{aligned}
$$

In a similar way, the expected profit $(\bar{U})$ after considering the demand distribution changes as follows:

$$
\begin{aligned}
\bar{U}= & \int_{d_{\min }}^{Q S} U_{1}\left(\frac{1}{\left(d_{\max }-d_{\min }\right)}\right) d(D)+\int_{Q S}^{Q S+\frac{Q R}{\alpha(1+\beta)}} \\
& U_{2}\left(\frac{1}{\left(d_{\max }-d_{\min }\right)}\right) d(D)+\int_{Q S+\frac{Q R}{\alpha(1+\beta)}}^{d_{\max }} \\
& U_{3}\left(\frac{1}{\left(d_{\max }-d_{\min }\right)}\right) d(D) .
\end{aligned}
$$

With the assumption of these objective functions, the mathematical model of the problem is as follows:

$$
\operatorname{Max} \overline{S L}
$$

$\operatorname{Max} \bar{U}$

s.t.:

$$
\begin{aligned}
& \sum_{i=1}^{n} C_{i}+\left(C^{\prime}+\sum_{i=1}^{n} C_{i}\right)(1+\beta) Q S \leq B \\
& \sum_{i=1}^{n} V R_{i} Q R+V S Q S \leq I \\
& Q R, Q S \leq d_{\max } \\
& Q S \geq d_{\min } \\
& Q R \geq 0 .
\end{aligned}
$$

Eq. (16) refers to the budget constraint before the beginning of the period. The total budget used for purchasing raw materials and producing the initial inventory of the finished product is limited. Eq. (17) is the storage space constraint. The space assigned to the initial inventory of raw materials and the finished product is limited.

\section{Solution approach}

In the previous section, the problem and its mathematical model are discussed. Since our problem has two objective functions, by ignoring each of the objective functions, two single-objective function problems can be obtained: Presenting the way of solving the problem in the case of the single-objective function and discussing the way of solving the problem with the integrated objective functions are as follow.

In the first step for solving two single-objective problems, we have two nonlinear objective functions that should be maximized in a feasible region created by some constraints. Upon reviewing the feasible region of these problems, we obtain that the variable $Q S$ takes limited positive and nonzero values, and the variable $Q R$ takes limited positive values. Therefore, in the first problem, the function $\overline{S L}$ that is defined in Eq. (12) is continuous and has the first partial derivatives in this feasible region. In the second problem, the objective function $(\bar{U})$ that is expressed in Eq. (13) consists of variables $U_{1}, U_{2}$, and $U_{3}$. Variables $U_{1}, U_{2}$, and $U_{3}$ defined in Eqs. (2), (4), and (6) are polynomial functions of variables $Q S, Q R$, and $D$. In this respect, the expected profit $(\bar{U})$ in Eq. (13) is a polynomial function. Consequently, the objective function in the second problem is also continuous and has first partial derivatives in the feasible region. In this respect, in both of the problems, objective functions are continuous and have the first partial derivatives.

In order to solve these two nonlinear problems, the SUMT technique that is known as one of the best methods for constrained nonlinear problems is 
employed. This method uses some penalty functions for transforming the constrained nonlinear problem into a sequence of unconstrained problems [29]. In this technique, instead of each constraint, a term is added to the objective function as a function of that constraint. In doing so, the constrained nonlinear problem is transformed into a non-constrained nonlinear problem. In fact, by these changes, the resulting objective function is penalized whenever these constraints are violated. The only necessary condition for applying this method is the continuity of the objective functions and constraints [29-31]; this necessary condition fits well with our two single-objective problems. The transformed problems are then solved using a suitable technique for manipulating the unconstrained nonlinear problem.

Boukari and Fiacco [29] discussed various penalty functions. In this paper, we used the inverse penalty function as one of the most popular penalty functions to transform our constrained model to an unconstrained one. This function works as follows:

Original problem:

Min $f(x)$,

Inverted problem:

$$
\operatorname{Min} \operatorname{Inv}\left(x, r_{k}\right)=f(x)+r_{k} \sum_{i=1}^{m} \frac{1}{g_{i}(x)},
$$

s.t.:

$$
g_{i}(x) \geq 0, \quad i=1,2, \ldots, m .
$$

In the inverted model, $r_{k}>0$ is a real number whose value decreases sequentially depending on the number of iterations of using the SUMT technique.

In order to use the SUMT technique, the objective functions are written in a minimum form and the constraints are expressed in nonnegative forms as follows:

$$
\begin{aligned}
& \operatorname{Min}-\overline{S L}, \\
& \operatorname{Min}-\bar{U},
\end{aligned}
$$

s.t.:

$$
g_{1}(Q R, Q S): \quad B-\sum_{i=1}^{n} C_{i}-\left(C^{\prime}+\sum_{i=1}^{n} C_{i}\right)
$$$$
(1+\beta) Q S \geq 0,
$$

$$
\begin{array}{ll}
g_{2}(Q R, Q S): & I-\sum_{i=1}^{n} V R_{i} Q R-V S Q S \geq 0 \\
g_{3}(Q R, Q S): & d_{\max }-Q R \geq 0 \\
g_{4}(Q R, Q S): & d_{\max }-Q S \geq 0 \\
g_{5}(Q R, Q S): & Q S-d_{\min } \geq 0 \\
g_{6}(Q R, Q S): & Q R \geq 0
\end{array}
$$

Using the inverse penalty function, we obtain the following unconstrained problems:

Problem 1:

$$
\text { Min } F_{1}=-\overline{S L}+\text { penalty }_{k} \sum_{i=1}^{6} \frac{1}{g_{i}(Q R, Q S)},
$$

Problem 2:

$$
\text { Min } F_{2}=-\bar{U}+\text { penalty }_{k} \sum_{i=1}^{6} \frac{1}{g_{i}(Q R, Q S)} .
$$

We suppose that penalty ${ }_{k}>0$ is a real number, which takes the value one in the first iteration (penalty $y_{k}=$ $1, k=1$ ) and decreases sequentially by being multiplied by the value of 0.1 in each iteration.

As already mentioned, $\overline{S L}$ and $\bar{U}$ are both continuous and have the first partial derivatives in the feasible region. In addition, all $g_{i}(Q R, Q S) ; i=1,2, \ldots, 6$ are linear functions. As a result, $F_{1}$ and $F_{2}$ have continuous first partial derivatives except in the boundary of the feasible region. In this respect, the SD technique can be selected among various methods of unconstrained nonlinear optimization to solve our two single-objective problems.

The SD technique is based on the first derivatives and is one of the fundamental techniques for minimizing a differentiable function with multiple variables. The main idea behind SD is that if $f(x)$ is a function of $x$ with a nonzero gradient at $x$, then by moving from $x$ in the direction $\frac{-\nabla f(x)}{\|\nabla f(x)\|}$, we will find the next point at which the function is of the highest decreasing value. This iterative improvement technique is repeated until no significant improvement in the objective function is observed [30,32].

To determine the minimum value of the objective function $F(Q R, Q S)$ using the SD method, we start from $X$ as an arbitrary point in the feasible region and proceed through some sequential iterations using the following formula:

$$
X_{k+1}=X_{k}-t . g r\left(X_{k}\right),
$$

where parameter $k$ is the number of iterations. Function $\operatorname{gr}\left(X_{k}\right)$ is the gradient of $F(Q R, Q S)$ at the point $X_{k}$ and parameter $t$ is a scalar value determined by solving the equation $\frac{d}{d t} X_{k+1}=0$ at each iteration. After that, the next point $X_{k+1}$ is calculated using Eq. (30) and in this way, the improvement of the objective function is made at each iteration. This procedure is repeated until the values of the objective function do not significantly improve between two sequential iterations.

Up to now, the initial problem is considered as two various single-objective nonlinear problems. Both of these problems are optimized separately by mixing SUMT and SD techniques. In the following, we discuss 
how to consider a new single-objective function instead of our initial two objective functions. Moreover, this new problem can again be solved by mixing SUMT and SD techniques.

In order to optimize our initial problem with two objective functions, we utilize the Multi-Objective Optimization (MOO) techniques. Pareto and scalarization are two methods of the MOO techniques. Pareto method uses a continuously updated algorithm to determine dominated and non-dominated solutions. Meanwhile, the scalarization method uses some weights for objective functions and finally, converts the MOO problem into a single-objective optimization problem [33]. This study employs the global criterion method, which is one of the most common scalarization methods. The global criterion is a scalar function obtained by a mathematical combination of initial multiobjective functions [34]. This scalar function is used to minimize the distance between multiple reference points (ideal solutions) and viable destination areas [33].

By utilizing the global criterion method with the following illustration, we actually work with a singleobjective optimization problem instead of our initial MOO:

Multi-objective form:

$$
\operatorname{Max} f_{j j}(x) ; \quad j j=1, \ldots, k .
$$

The equivalent single-objective form:

$$
\operatorname{Min} l_{p}=\left\{\sum_{j j=1}^{k} w_{j j}\left(\frac{\left(f_{j j}\left(x^{*}\right)-f_{j j}(x)\right)}{f_{j j}\left(x^{*}\right)}\right)^{p}\right\}^{\frac{1}{p}},
$$

where $f_{j j}\left(x^{*}\right)$ represents the maximum value of the function $f_{j j}\left(x^{*}\right)$ and $w_{j j}$ is a non-negative weight that points to the importance of the corresponding objective function. The value $p$ is a distance metric. Then, each of the terms of the objective functions is divided by $f_{j j}\left(x^{*}\right)$, resulting in the removal of the effect of different measurement scales from the objective functions $[34,35]$.
Eq. (32) illustrates the result of using the global criterion method for integrating our objective functions:

$$
\begin{aligned}
\operatorname{Min} l_{p}= & \left\{w_{1}\left(\frac{\left(f_{1}\left(x_{1}^{\max }\right)-f_{1}(x)\right)}{f_{1}\left(x_{1}^{\max }\right)}\right)^{p}\right. \\
& \left.+w_{2}\left(\frac{\left(f_{2}\left(x_{2}^{\max }\right)-f_{2}(x)\right)}{f_{2}\left(x_{2}^{\max }\right)}\right)^{p}\right\}^{\frac{1}{p}},
\end{aligned}
$$

where $f_{1}(x)$ is the first objective function (i.e., the expected service level $\overline{S L}$ in Eq. (12)), and $f_{1}\left(x_{1}^{\max }\right)$ is the maximum amount of $\overline{S L}$ determined by assuming that it is the only objective function of the study. In a similar way, $f_{2}(x)$ corresponds to the expected total profit, $\bar{U}$, in Eq. (13), and $f_{2}\left(x_{2}^{\max }\right)$ represents the maximum amount of $\bar{U}$ by assuming that it is the only objective function. The multipliers $w_{1}$ and $w_{2}$ are scalar values that point to the importance of each objective function. Parameter $p$ denotes the metric. We consider the Manhattan metric $(p=1)$. In Eq. (13), $\overline{S L}$ is the expected service level and takes its value at the interval $(0,100)$, but $\bar{U}$ is a monetary value (i.e., the earned profit). The divisions in Eq. (31) are meant to eliminate the effect of these two different scales.

In our initial model, the objective function $\min l_{p}$ replaces the two objective functions $\overline{S L}, \bar{U}$, and the constraints are $g_{i}(Q R, Q S) i=1,2, \ldots, 6$, which are formulated in Eqs. (23) to (27). Again, because the necessary conditions of the SUMT and SD techniques remained unchanged in this new problem, these techniques are applied for solving the new problem. The next section illustrates a sample problem and its solution results follow this approach.

\section{Computational results}

\subsection{The sample problem}

The sample problem considered in this section has the following descriptions: A limited budget, $B=$ $15,000,000$ units of money, is available before the beginning of the period, and the total available storage space is $I=100,000$ units. The finished product is made of four different raw materials whose specifications are given in Table 2. The demand value $D$ during

Table 2. Specifications of raw materials.

\begin{tabular}{ccccc}
\hline $\begin{array}{c}\text { Raw } \\
\text { materials }\end{array}$ & $\begin{array}{c}\text { Purchasing price } \\
\text { per unit }\end{array}$ & $\begin{array}{c}\text { Selling price } \\
\text { per unit after } \\
\text { the period }\end{array}$ & $\begin{array}{c}\text { Holding cost } \\
\text { per unit }\end{array}$ & $\begin{array}{c}\text { Volume per } \\
\text { unit }\end{array}$ \\
\hline I & $\boldsymbol{C}_{\boldsymbol{i}}$ & $\boldsymbol{L}_{\boldsymbol{i}}$ & $\boldsymbol{h}_{\boldsymbol{i}}$ & $\boldsymbol{V R}_{\boldsymbol{i}}$ \\
\hline 1 & 500 & 450 & 125 & 21 \\
2 & 420 & 400 & 210 & 26 \\
3 & 810 & 800 & 170 & 23 \\
4 & 750 & 700 & 195 & 24 \\
\hline
\end{tabular}


Table 3. Specifications of the finished product.

\begin{tabular}{lcc}
\hline The selling price per unit during the period & $R$ & 90,000 \\
The selling price per unit of wastages & $L w$ & 10,000 \\
The cost of production per unit & $C^{\prime}$ & 40,000 \\
The volume per unit & $V S$ & 200 \\
The cost of holding per unit & $H$ & 20,000 \\
The selling price per unit after the period & $L^{\prime}$ & 50,000 \\
The cost of lost demand per unit & $\pi$ & 60,000 \\
\hline
\end{tabular}

the period is a random variable at the interval $(300$, $1000)\left(\right.$ or $\left.d_{\min }=300, d_{\max }=1000\right)$ with a continuous uniform distribution.

The production of each unit of the finished product consumes 40,000 units of money, that is, one unit from each of the four raw materials. The production process has a wastage of $5 \%(\beta=0.05)$, and $40 \%$ of the customers can wait for their demand to be fulfilled $(\alpha=0.4)$. An overview of the finished products is presented in Table 3.

This sample problem is solved using the approach presented in the last section and proper coding in MATLAB (2013) on a laptop equipped with Intel Core i7-processor and 8 GB of RAM under Windows 8.1 operating system.

The details of the solution method and its result are presented as follows: The point $(Q S=200, Q R=$ $200)$ is arbitrarily selected as the initial feasible point.

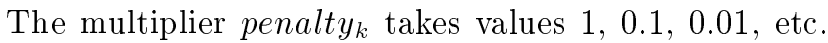
at 10 iterations. These multiplier values for the inverse penalty function of the constraints are added to the objective function in the SUMT technique. At each iteration of the SUMT, the resulting unconstrained objective function is solved through the SD procedure at seven iterations.

In the first step, each of the objective functions is considered separately. When $\overline{S L}$ is assumed to be the only objective function of the study, the optimal value of $90.1 \%$ is obtained for $\overline{S L}$ at the optimal point $(Q S=335.77, Q R=329.94)$. That is, by setting the levels of inventory of raw material and finished products as $(Q S=335.77, Q R=329.94)$, the best level of satisfying the demands is expected to be $90.1 \%$. On the other hand, when $\bar{U}$ is the only objective function, we get the optimal value of $9,416,684.5$ for $\bar{U}$ at the optimal point $(Q S=331.37$, $Q R=249.21)$. In other words, the best value of the profit is expected to be $9,416,684.5$ units of money and for reaching this amount, the levels of inventory should be set as $(Q S=331.37, Q R=249.21)$. In both of the problems, it is observed that after a few iterations of SUMT execution, all the values remain the same. More details are given in Tables 4 and 5. In fact, after running one iteration of SUMT, which includes seven iterations of SD, the last result is almost obtained. In

Table 4. A summary of calculations with the expected service level $(\overline{S L})$ as the only objective function.

\begin{tabular}{ccccc}
\hline No. of iterations & QS & QR & $\overline{\boldsymbol{S L}}$ & $\overline{\boldsymbol{U}}$ \\
\hline 1 & 200 & 200 & 65.47 & $-2,124,056.6$ \\
2 & 335.7688 & 329.9381 & 90.09 & $8,934,258.4$ \\
3 & 335.7688 & 329.9381 & 90.10 & $8,934,258.4$ \\
4 & 335.7688 & 329.9381 & 90.10 & $8,934,258.4$ \\
5 & 335.7688 & 329.9381 & 90.10 & $8,934,258.4$ \\
6 & 335.7688 & 329.9381 & 90.10 & $8,934,258.4$ \\
7 & 335.7688 & 329.9381 & 90.10 & $8,934,258.4$ \\
8 & 335.7688 & 329.9381 & 90.10 & $8,934,258.4$ \\
9 & 335.7688 & 329.9381 & 90.10 & $8,934,258.4$ \\
10 & 335.7688 & 329.9381 & 90.10 & $8,934,258.4$ \\
\hline
\end{tabular}

Table 5. A summary of calculations with the expected profit $(\bar{U})$ as the only objective function.

\begin{tabular}{ccccc}
\hline No. of iterations & QS & QR & $\overline{\boldsymbol{S L}}$ & $\overline{\boldsymbol{U}}$ \\
\hline 1 & 200 & 200 & 65.49 & $-2,124,056.64$ \\
2 & 331.3736 & 249.2057 & 84.33 & $9,416,684.46$ \\
3 & 331.3736 & 249.2057 & 84.33 & $9,416,684.46$ \\
4 & 331.3736 & 249.2057 & 84.33 & $9,416,684.47$ \\
5 & 331.3736 & 249.2057 & 84.33 & $9,416,684.47$ \\
6 & 331.3736 & 249.2057 & 84.33 & $9,416,684.47$ \\
7 & 331.3736 & 249.2057 & 84.33 & $9,416,684.47$ \\
8 & 331.3736 & 249.2057 & 84.33 & $9,416,684.47$ \\
9 & 331.3736 & 249.2057 & 84.33 & $9,416,684.47$ \\
10 & 331.3736 & 249.2057 & 84.33 & $9,416,684.47$ \\
\hline
\end{tabular}


Table 6. A summary of calculations with the aggregated objective (LP) as the only objective function when starting at the arbitrary point $(Q S=200, Q R=200)$.

\begin{tabular}{cccccc}
\hline No. of iterations & QS & QR & LP & $\overline{\boldsymbol{S L}}$ & $\overline{\boldsymbol{U}}$ \\
\hline 1 & 200.00 & 200.00 & $1,061,908.37$ & 65.49 & $-2,124,056.62$ \\
2 & 305.97 & 241.96 & $-3,843,317.18$ & 81.59 & $7,686,378.33$ \\
3 & 305.97 & 241.96 & $-3,843,317.18$ & 81.59 & $7,686,378.33$ \\
4 & 305.97 & 241.96 & $-3,843,317.18$ & 81.59 & $7,686,378.33$ \\
5 & 305.97 & 241.96 & $-3,843,317.18$ & 81.59 & $7,686,378.33$ \\
6 & 305.97 & 241.96 & $-3,843,317.18$ & 81.59 & $7,686,378.33$ \\
7 & 305.97 & 241.96 & $-3,843,317.18$ & 81.59 & $7,686,378.33$ \\
8 & 305.97 & 241.96 & $-3,843,317.18$ & 81.59 & $7,686,378.33$ \\
9 & 305.97 & 241.96 & $-3,843,317.18$ & 81.59 & $7,686,378.33$ \\
10 & 305.97 & 241.96 & $-3,843,317.18$ & 81.59 & $7,686,378.33$ \\
\hline
\end{tabular}

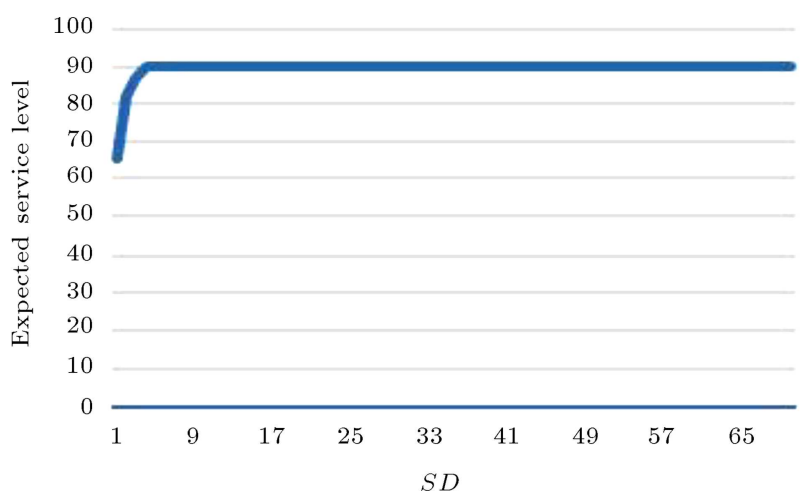

Figure 1. Convergence plot of the service level $\overline{S L}$ by running the Steepest Descent (SD) (each Sequential Unconstrained Minimization Technique (SUMT) iteration equivalent to seven iterations of SD).

Figures 1 and 2, convergence to the optimum point based on iterations of SD is illustrated. As mentioned earlier, each iteration of SUMT technique includes seven iterations of SD technique. As can be observed, upon running the initial iterations of SD technique in the first iteration of SUMT, the plot reaches its equilibrium state.

The global criterion method is employed based on Eq. (31) to aggregate the objective functions. The resulting objective function with the same set of constraints is optimized via the SUMT and SD techniques starting at the arbitrary point $(Q S=200$, $Q R=200)$. We assume the same weights $w_{1}=0.5$ and $w_{2}=0.5$, suggesting the equal importance of the objective functions. In addition, the parameter $p=1$ is considered for Manhattan distance. The optimal point $(Q S=305.97, Q R=241.96)$ is obtained, and it is expected that about $81.6 \%$ of demand will be fulfilled and about 7,686,381.9 units of monetary profit be earned at this point. A summary of the results is given in Table 6 . Here again, we can observe that after

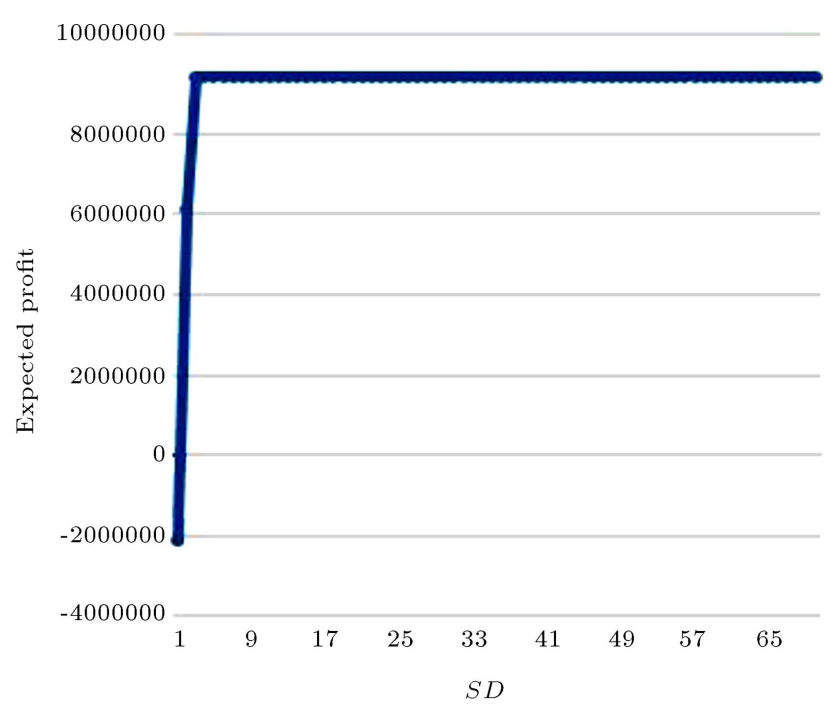

Figure 2. Convergence plot of the expected profit $\bar{U}$ by running the Steepest Descent (SD) (each Sequential Unconstrained Minimization Technique (SUMT) iteration equivalent to seven iterations of SD).

running one iteration of the SUMT, the last result is obtained. In Figure 3, convergence to the optimum point based on iterations of the SD is illustrated. As can be seen, just after running the initial iterations of the SD technique in the first iteration of SUMT, the plot reaches its equilibrium state.

The aggregate objective function $L_{p}$ described in the previous section with the same parameter values is again solved using a different starting point ( $Q S=$ $335.77, Q R=329.94)$, which is the optimal point of the problem when $\overline{S L}$ (the expected service level) is assumed as the only objective function. The results are summarized in Table 7 . As seen, the value of the optimal objective function does not point to any significant improvement through the 10 iterations.

Again, the aggregate objective function LP 
Table 7. A summary of calculations with the aggregated objective (LP) as the only objective function when starting at the point $(Q S=335.77, Q R=329.94)$ (obtained from Table 4$)$.

\begin{tabular}{cccccc}
\hline No. of iterations & QS & QR & LP & $\overline{\boldsymbol{S L}}$ & $\overline{\boldsymbol{U}}$ \\
\hline 1 & 335.77 & 329.94 & $-4,467,267.40$ & 90.10 & $8,934,270.27$ \\
2 & 335.77 & 329.94 & $-4,467,267.41$ & 90.10 & $8,934,270.27$ \\
3 & 335.77 & 329.94 & $-4,467,267.41$ & 90.10 & $8,934,270.27$ \\
4 & 335.77 & 329.94 & $-4,467,267.41$ & 90.10 & $8,934,270.27$ \\
5 & 335.77 & 329.94 & $-4,467,267.41$ & 90.10 & $8,934,270.27$ \\
6 & 335.77 & 329.94 & $-4,467,267.41$ & 90.10 & $8,934,270.27$ \\
7 & 335.77 & 329.94 & $-4,467,267.41$ & 90.10 & $8,934,270.27$ \\
8 & 335.77 & 329.94 & $-4,467,267.41$ & 90.10 & $8,934,270.27$ \\
9 & 335.77 & 329.94 & $-4,467,267.41$ & 90.10 & $8,934,270.27$ \\
10 & 335.77 & 329.94 & $-4,467,267.41$ & 90.10 & $8,934,270.27$ \\
\hline
\end{tabular}

Table 8. A summary of calculations with the aggregated objective (LP) as the only objective function when starting at the point $(Q S=331.37, Q R=249.21)$ (obtained from Table 4$)$.

\begin{tabular}{cccccc}
\hline No. of iterations & QS & QR & LP & $\overline{\boldsymbol{S L}}$ & $\overline{\boldsymbol{U}}$ \\
\hline 1 & 331.37 & 249.21 & $-4,708,369.60$ & 84.33 & $9,416,480.47$ \\
2 & 331.37 & 249.21 & $-4,708,369.61$ & 84.33 & $9,416,480.47$ \\
3 & 331.37 & 249.21 & $-4,708,369.61$ & 84.33 & $9,416,480.47$ \\
4 & 331.37 & 249.21 & $-4,708,369.61$ & 84.33 & $9,416,480.47$ \\
5 & 331.37 & 249.21 & $-4,708,369.61$ & 84.33 & $9,416,480.47$ \\
6 & 331.37 & 249.21 & $-4,708,369.61$ & 84.33 & $9,416,480.47$ \\
7 & 331.37 & 249.21 & $-4,708,369.61$ & 84.33 & $9,416,480.47$ \\
8 & 331.37 & 249.21 & $-4,708,369.61$ & 84.33 & $9,416,480.47$ \\
9 & 331.37 & 249.21 & $-4,708,369.61$ & 84.33 & $9,416,480.47$ \\
10 & 331.37 & 249.21 & $-4,708,369.61$ & 84.33 & $9,416,480.47$ \\
\hline
\end{tabular}

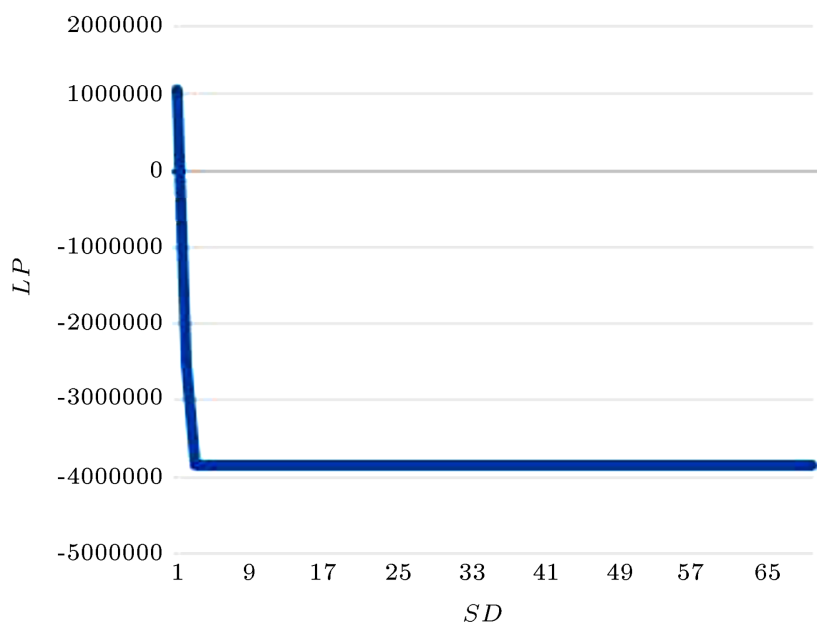

Figure 3. Convergence plot of the aggregated objective $L_{p}$ by iterations of the Steepest Descent (SD) (each Sequential Unconstrained Minimization Technique (SUMT) iteration equivalent to seven iterations of SD).

is solved one more time using the same parameter values, but starting at a new point $(Q S=331.37$, $Q R=249.21)$ obtained as the optimal point when $\bar{U}$ (the expected profit) is assumed the only objective function. According to Table 8, the value of the optimal objective function does not improve significantly throughout the 10 iterations.

Comparison of the results of the last three tables (Tables 6-8) illustrates that the two previous ones give better results. Alternatively, the better results come from starting with the optimal points of the problems with the single objective than from starting with our initial arbitrary feasible point. To evaluate the starting points of single-objective problems by comparing the values of $L_{p}$, it can be concluded that Table 8 is the best because of the smaller $L_{p}$. In other words, in the case of this problem, the optimal objectives obtain the expected profit, i.e., 9,416,480.47 units of money, and the expected completion of $84.33 \%$ of demands. In order to reach these results, it is necessary to set the inventory levels of finished product and raw materials as $(Q S=331.37, Q R=249.21)$ before the start of the selling period.

\subsection{Verification, validation, and sensitivity analysis}

In order to ensure the quality of the implemented method, some tests are done to check if the method runs properly. Verification of the method or assurance about building the right model is controlled by debug- 
ging and tracing the program code of the mathematical model. Validation of the method or assurance about building the right model is controlled by examining the results of about 50 sample tests. In each of these tests, we select a starting point arbitrarily and calculate the value of the objective function. Then, after running the method with this starting point, we again calculate the value of the objective function at the final point. A comparison between the values of the objective function points to its improvement upon running the code of the method. The validity of the results of these tests is also managed manually, which is in agreement with the results obtained through the computer code. Of note, similar to all other exact nonlinear programming methods, the final point is completely related to the selection of the starting point and by varying the start point, the final point is changed.

The sensitivity analysis of the solution approach based on parameters $w_{1}, w_{2}$, and penalty $y_{k}$ is examined. In all these tests, it is supposed that each iteration of the SUMT calls seven times the SD and in all the tests, it starts at point $(Q S=200, Q R=200)$.

In the first step, we only change the parameters $w_{1}, w_{2}$ or weights of two objective components. In this test, similar to the previous part, the SUMT function runs 10 times $(k=1,2, \ldots, 10)$ or the value of penalty $_{k}=1, k=1$ multiplies by 0.1 in iterations of running SUMT function. Table 9, Figures 4 and 5 illustrate the variation of objective components by changing the weights. These figures show that the changes in the objective components are very small.

In the second step, we set three different settings for parameter penalty :

- SUMT function runs 5 times $(k=1,2, \ldots, 5)$ or the value of penalty $_{k}=1, k=1$ multiplies by 0.2 in each iteration of function SUMT;

- SUMT function runs 10 times $(k=1,2, \ldots, 10)$ or the value of penalty $_{k}=1, k=1$ multiplies by 0.1 in each iteration of function SUMT;

- SUMT function runs 20 times $(k=1,2, \ldots 20)$ or the value of penalty $_{k}=1, k=1$ multiplies by 0.05 in each iteration of function SUMT.

Then, these settings are considered to solve the problem with only one objective function and again,

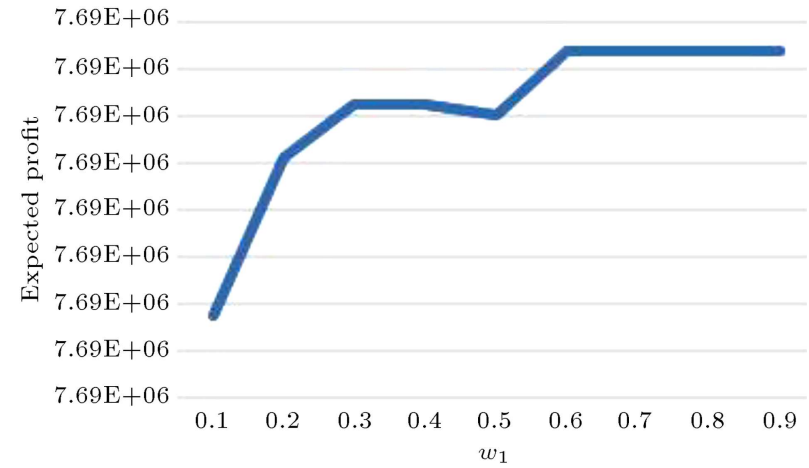

Figure 4. Changes of the expected profit $\bar{U}$ by varying the weight $w_{1}$.

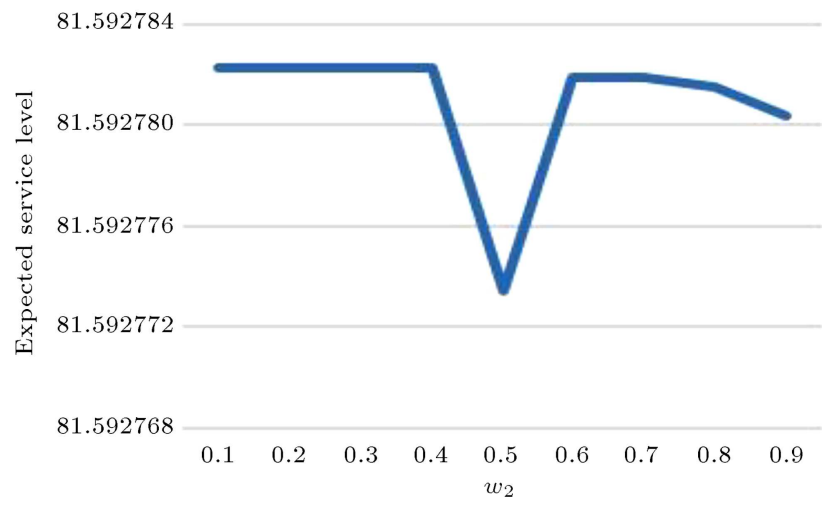

Figure 5. Changes of the service level $\overline{S L}$ by varying the weight $w_{2}$.

the problem with two objective functions and weights $w_{1}=0.5$, and $w_{2}=0.5$.

Tables 10-12 and Figures 6-8 show the results of these tests when SUMT functions run 5 times. As can be seen in these tables and figures, in all of them in the first iteration of function SUMT and in the second or third iteration on function SD, the proposed approach reaches the final solution. In other iterations, this solution cannot be enhanced. The same result with the same final solution is obtained upon repeating the test by setting the run number of SUMT function to 10 and 20 (changing the settings of penalty $y_{k}$ ). The results show that after the first iteration of the SUMT, the method almost reaches its optimum point and it is not sensitive to penalty $y_{k}$.

Table 9. Sensitivity analysis of the solution approach by varying the weights.

\begin{tabular}{cccccccccc}
\hline $\boldsymbol{w}_{\mathbf{1}}$ & 0.1 & 0.2 & 0.3 & 0.4 & 0.5 & 0.6 & 0.7 & 0.8 & 0.9 \\
$\boldsymbol{w}_{\boldsymbol{2}}$ & 0.9 & 0.8 & 0.7 & 0.6 & 0.5 & 0.4 & 0.3 & 0.2 & 0.1 \\
$\boldsymbol{Q S}$ & 305.97 & 305.97 & 305.97 & 305.97 & 305.97 & 305.97 & 305.97 & 305.97 & 305.97 \\
$\boldsymbol{Q R}$ & 241.96 & 241.96 & 241.96 & 241.96 & 241.96 & 241.96 & 241.96 & 241.96 & 241.96 \\
$\overline{\boldsymbol{S L}}$ & 81.59 & 81.59 & 81.59 & 81.59 & 81.59 & 81.59 & 81.59 & 81.59 & 81.59 \\
$\overline{\boldsymbol{U}}$ & 7686353.67 & 7686370.69 & 7686376.36 & 7686376.36 & 7686375.17 & 7686382.03 & 7686382.03 & 7686382.03 & 7686382.03 \\
\hline
\end{tabular}


Table 10. Changes of the optimum point of the aggregated objective (LP) in various iterations of Steepest Descent (SD) when Sequential Unconstrained Minimization Technique (SUMT) runs 5 times (penalty $y_{k}$ times (penalty $y_{k}$ Technique (UMT

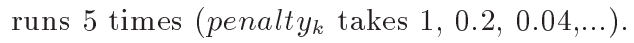

\begin{tabular}{|c|c|c|c|c|c|c|c|c|c|c|c|}
\hline NO. SUMT & & & & 1 & & & & 2 & 3 & 4 & 5 \\
\hline NO. SD & 1 & 2 & 3 & 4 & 5 & 6 & 7 & $8-14$ & $15-21$ & $22-28$ & $29-35$ \\
\hline QS & 200.00 & 271.23 & 305.97 & 305.97 & 305.97 & 305.97 & 305.97 & 305.97 & 305.97 & 305.97 & 305.97 \\
\hline QR & 200.00 & 232.57 & 241.96 & 241.96 & 241.96 & 241.96 & 241.96 & 241.96 & 241.96 & 241.96 & 241.96 \\
\hline $\mathbf{L P}$ & -1061908 & -2507436 & -3843317 & -3843317 & -3843317 & -3843317 & -3843317 & -3843317 & -3843317 & -3843317 & -3843317 \\
\hline
\end{tabular}

Table 11. Changes of the optimum point of the expected profit $(\bar{U})$ in various iterations of Steepest Descent (SD) when

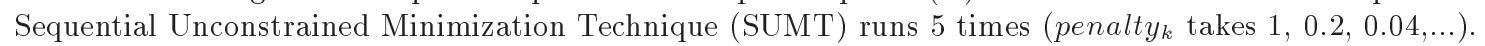

\begin{tabular}{|c|c|c|c|c|c|c|c|c|c|c|c|}
\hline NO. SUMT & & & & 1 & & & & 2 & 3 & 4 & 5 \\
\hline NO. SD & 1 & 2 & 3 & 4 & 5 & 6 & 7 & $8-14$ & $15-21$ & $22-28$ & $29-35$ \\
\hline QS & 200.00 & 284.78 & 331.37 & 331.37 & 331.37 & 331.37 & 331.37 & 331.37 & 331.37 & 331.37 & 331.37 \\
\hline QR & 200.00 & 238.76 & 249.21 & 249.21 & 249.21 & 249.21 & 249.21 & 249.21 & 249.21 & 249.21 & 249.21 \\
\hline $\bar{U}$ & -2124057 & 6141625 & 9416684 & 9416684 & 9416684 & 9416684 & 9416684 & 9416684 & 9416684 & 9416684 & 9416684 \\
\hline
\end{tabular}

Table 12. Changes of the optimal point of the expected service level $(\overline{S L})$ in various iterations of Steepest Descent (SD) when Sequential Unconstrained Minimization Technique (SUMT) runs 5 times (penalty $y_{k}$ takes $1,0.2,0.04, \ldots$ ).

\begin{tabular}{lcccccccccccc}
\hline NO. SUMT & & & \multicolumn{1}{c}{1} & \multicolumn{3}{c}{3} & \multicolumn{2}{c}{3} & \multicolumn{2}{c}{3} \\
\hline NO. SD & 1 & 2 & 3 & 4 & 5 & 6 & 7 & $8-14$ & $15-21$ & $22-28$ & $29-35$ \\
\hline QS & 200.00 & 279.73 & 309.62 & 335.77 & 335.77 & 335.77 & 335.77 & 335.77 & 335.77 & 335.77 & 335.77 \\
QR & 200.00 & 276.28 & 304.90 & 329.94 & 329.94 & 329.94 & 329.94 & 329.94 & 329.94 & 329.94 & 329.94 \\
$\overline{S L}$ & 65.47 & 82.16 & 86.74 & 90.09 & 90.09 & 90.09 & 90.09 & 90.09 & 90.09 & 90.09 & 90.09 \\
\hline
\end{tabular}

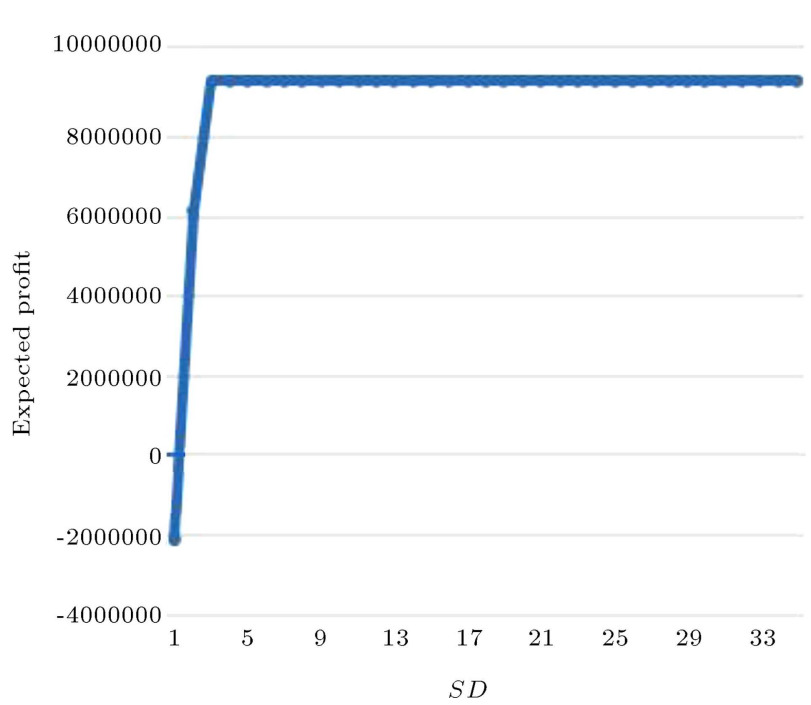

Figure 6. Changes of the expected profit $\bar{U}$ in various iterations of Steepest Descent (SD) when Sequential Unconstrained Minimization Technique (SUMT) runs 5 times.

\section{Conclusion and future works}

In this paper, a two-echelon newsvendor model (NVM) with two objective functions, i.e., service level and total profit, was modeled. The study constraints were budget and storage space. The main problem was the nonlinear problem with two objective functions and some constraints. In the first step, two single-objective problems were determined regardless of each initial

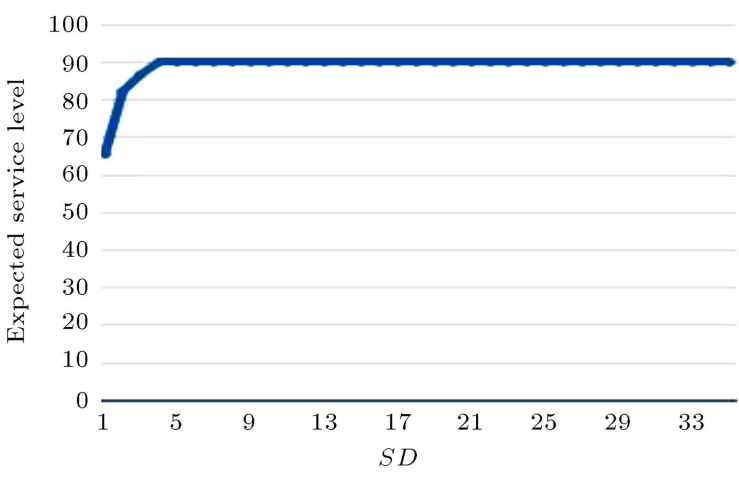

Figure 7. Changes of the service level $\overline{S L}$ in various iterations of Steepest Descent (SD) when Sequential Unconstrained Minimization Technique (SUMT) runs 5 times.

object function. Both of the problems had the necessary requirements for being resolved using Sequential Unconstrained Minimization Technique (SUMT) method. SUMT method transforms a constrained nonlinear problem to a series of unconstraint problems. These unconstrained nonlinear problems can be solved with Steepest Descent (SD) technique. In this way, the optimum points in each of these two single-objective problems were obtained. In order to solve the initial problem, the global criterion method that belonged to the scalarization method of Multi-Objective Optimization (MOO) methods was employed. Scalarization methods differed from Pareto methods. These methods assign weights to each objective function and, finally, 


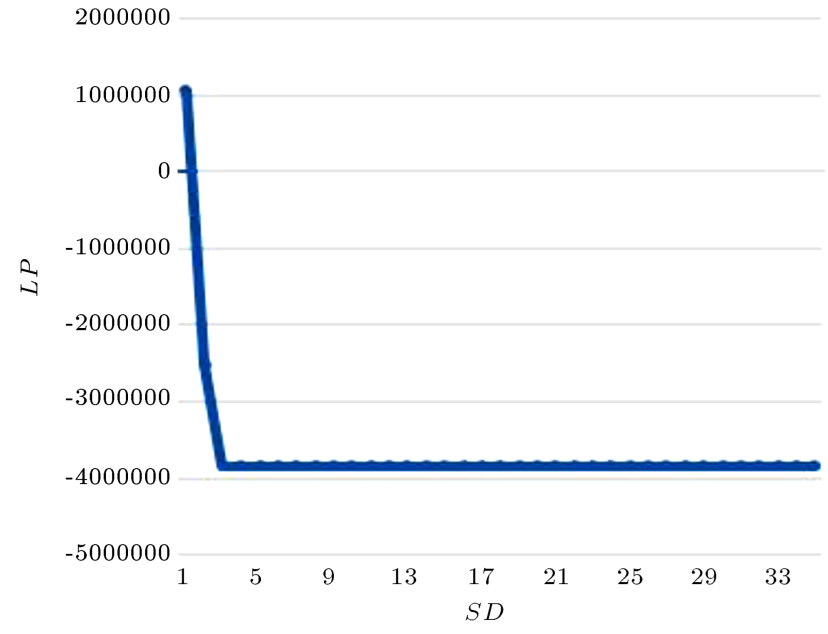

Figure 8. Changes of the aggregated objective LP in various iterations of Steepest Descent (SD) when Sequential Unconstrained Minimization Technique (SUMT) runs 5 times.

give one scalar objective function instead of the initial multi-objective functions. Global criterion method takes the optimum points of the two single-objective problems and two weights for each objective function and, finally, gives a single-objective function. This final problem was solved like the two earlier ones using a mixture of SUMT and SD methods. A numerical example was solved using a computer code developed on the MATLAB software.

Our main contribution in this paper is the application of the mixture of SUMT and SD techniques from exact methods in nonlinear programming for solving an NVM. This method is rarely used for solving the NVMs in the literature. Considering both goals of producer and consumer in the objective function is another contribution. In this problem, it is supposed that the values could take any real number and working on the integer version of this problem is proposed as future work. Moreover, we suppose that the demand has a predefined distribution which is a reasonable assumption for general products, whereas there is not any information on the demand for new products. As another future work, we suggest considering this problem when the demand is distribution free.

\section{References}

1. Qin, Y., Wang, R., Vakharia, A., et al. "The newsvendor problem: review and directions for future research", European Journal of Operational Research, 213(2), pp. 361-374 (2011).

2. Sariyer, G. "Sizing capacity levels in emergency medical services dispatch centers: Using the newsvendor approach", The American Journal of Emergency Medicine, 36(5), pp. 804-815 (2018).

3. Olivares, M., Terwiesch, C., and Cassorla, L. "Struc- tural estimation of the newsvendor model: an application to reserving operating room time", Management Science, 54(1), pp. 41-55 (2008).

4. Wachtel, R. and Dexter, F. "Review of behavioral operations experimental studies of newsvendor problems for operating room management", Anesthesia Analgesia, 110(6), pp. 1698-1710 (2010).

5. Abedini, A., Li, W., and Ye, H. "Stochastic bilevel optimization models for efficient operating room planning", Procedia Manufacturing, 26, pp. 58-69 (2018).

6. Khouja, B. "The single period (news-vendor) problem: literature review and suggestions", Omega, 27(5), pp. 537-553 (1999).

7. Sundar, D., Ravikumar, K., and Mahajan, S. "The distribution free newsboy problem with partial information", Int. J. Operational Research, 33(4), pp. 481496 (2018).

8. Subrata, M. "Newsvendor problem with clearance pricing", European Journal of Operational Research, 268, pp. 193-202 (2018).

9. Sainathan, A. and Groenevelt, H. "Vendor managed inventory contracts-coordinating the supply chain while looking from the vendor's perspective", European Journal of Operational Research, 272, pp. 249-260 (2019).

10. Tekin, M. and Özekici S. "Mean-variance newsvendor model with random supply and financial hedging", IE Transactions, 47(9), pp. 910-928 (2015).

11. Pal, B., Sankar Sana, S., and Chaudhuri, K. "A distribution-free newsvendor problem with nonlinear holding cost", International Journal of Systems Science, 46(7), pp. 1269-1277 (2015).

12. Shi, Y., Alwan, L., Tang, C., et al. "A newsvendor model with auto correlated demand under a timeconsistent dynamic CVaR measure", IISE Transactions, 51(6), pp. 653-671 (2019).

13. Wang, T., Atasu, A., and Kurtuluş, M. "A multi ordering newsvendor model with dynamic forecast evolution", Manufacturing \& Service Operations Management, 14(3), pp. 355-484 (2012).

14. Abrudan, L.C., Matei, M.C., and Abrudan, M.M. "Running a business through a sharing responsibilities (and profits) strategy", Proceedings of the 12th International Management Conference, 12(1), pp. 2838 (2018).

15. Bieniek, M. "Goal setting in the newsvendor problem with uniformly distributed demand", Multiple Criteria Decision Making, 13, pp. 91-102 (2018).

16. Kim, G., Wu, K., and Huang, E. "Optimal inventory control in a multi-period newsvendor problem with non-stationary demand", Advanced Engineering Informatics, 29(1), pp. 139-145 (2015).

17. Alwan, L.C., Xu, M., Yao, D., et al. "The dynamic newsvendor model with correlated demand", Decision Sciences, 47(1), pp. 11-30 (2016). 
18. Alwan, L.C. and WeiB, C.H. "INAR implementation of newsvendor model for serially dependent demand counts", International Journal of Production Research, 55(4), pp. 1085-1099 (2017).

19. Xu, X., Meng, Z., Ji, P., et al. "On the newsvendor model with conditional Value-at-Risk of opportunity loss", International Journal of Production Research, 54(8), pp. 2449-2458 (2016).

20. Khouja, M., Liu, X., and Zhou, J. "To sell or not to sell to an off-price retailer in the presence of strategic consumers", Omega, 90, 102002 (2020).

21. Dai, J. and Meng, W. "A risk-averse newsvendor model under marketing-dependency and price-dependency", International Journal of Production Economics, 160, pp. 220-229 (2015).

22. Pasandideh, S.H., Akhavan Niyaki, S.T., and Rashidi, R. "A tow-echelon single-period inventory control problem under budget constraint", International Journal of Advanced Manufacturing Technology, 56(9-12), pp. 1205-1214 (2011).

23. Guler, K., Korpeouglu, E., and Sen, A. "Newsvendor competition under asymmetric cost information", European Journal of Operational Research, 271(2), pp. 561-576 (2018).

24. Mohammadivojdan, R. and Geunes, J. "The newsvendor problem with capacitated suppliers and quantity discounts", European Journal of Operational Research, 271, pp. 109-119 (2018).

25. Zhang, Y., Yang, X., and Li, B. "Distribution-free solutions to the extended multi-period newsboy problem", AIMS, 13(2), pp. 633-647 (2017).

26. Adhikary, K., Roy, J., and Kar, S. "A distributionfree newsboy problem with fuzzy random demand", International Journal of Management Science and Engineering Management, 13(3), pp. 200-208 (2018).

27. Watt, R. and Vazquez, F. "An analysis of insurance in the newsboy problem", European Journal of Operational Research, 259(3), pp. 1064-1072 (2017).

28. Ghiami, Y. and Williams, T. "A two-echelon production-inventory model for deteriorating items with multiple buyers", International Journal of Production Economics, 159, pp. 233-240 (2015).

29. Boukari, D. and Fiacco, V. "Survey of penalty, exactpenalty and multiplier methods from 1968 to 1993", Optimization: A Journal of Mathematical Programming and Operations Research, 32(4), pp. 301-334 (1995).
30. Bazara, M., Sherali, H., and Shetty, C., Nonlinear Programming Theory and Algorithms, John Wiley Sons, Atlanta, Georgia (1979).

31. Peressini, A., Sullivan, F., and Uhi, J., The Mathematics of Nonlinear Programming, Undergraduate Texts in Mathematics, Springer-Verlag (1988).

32. Petrova, S. and Solov'ev, A. "The origin of the method of steepest descent", Historia Mathematica, 24(4), pp. 361-375 (1997).

33. Gunantara, N. "A review of multi-objective optimization: Methods and its applications", Cogent Engineering, 5, pp. 1-16 (2018).

34. Marler, R. and Arora, J. "Survey of multi-objective optimization methods for engineering", Struct. Multidisc. Optim., 26(6), pp. 369-395 (2004).

35. Branke, J., Deb, K., Miettinen, K., et al., Multiobjective Optimization: Interactive and Evolutionary Approaches, Springer Science \& Business Media (2008).

\section{Biographies}

Maryam Yazdi is a $\mathrm{PhD}$ candidate at the Department of Industrial Engineering, Faculty of Industrial and Mechanical Engineering, Qazvin Branch, Islamic Azad University, Qazvin, Iran. She received her BSc from Computer and IT Group of the Department of Electrical \& Computer Engineering, Isfahan University of Technology, Isfahan, Iran. She obtained her MSc in the Industrial \& Systems Engineering Faculty at Tarbiat Modares University, Tehran, Iran. Her research interests include scheduling, meta-heuristic algorithms, multi-objective optimization, and inventory management.

Seyed Hamid Reza Pasandideh is an Associate Professor at the Department of Industrial Engineering at the Kharazmi University, Tehran, Iran. He received his BSc, MSc, and PhD in Industrial Engineering from Sharif University of Technology, Tehran, Iran. Also, he conducted postdoctoral research on cold supply chain at the University of Nebraska-Lincoln, Lincoln, US. His research interests include optimizing inventory control, multi-objective optimization, and application of queuing theory. He has published widely in those fields and he is the editor of some journals such as International Journal of Supply and Operations Management (IJSOM). 